\title{
On the Comparison and Evaluation of Some Basic Regulator Design Methods
}

\author{
László Keviczky, Csilla Bányász \\ Institute of Computer Science and Control, Systems and Control Laboratory, Budapest, Hungary
}

\section{Email address:}

keviczky@sztaki.hu (L. Keviczky), banyasz@sztaki.hu (C. Bányász)

\section{To cite this article:}

László Keviczky, Csilla Bányász. On the Comparison and Evaluation of Some Basic Regulator Design Methods. American Journal of Computer Science and Technology. Vol. 4, No. 3, 2021, pp. 83-89. doi: 10.11648/j.ajcst.20210403.14

Received: July 5, 2021; Accepted: September 2, 2021; Published: September 142021

\begin{abstract}
All existing basic regulator design methods are summarized in this paper and compared concerning their usability and formal algebraic formulations. First the regulators based on state-feedback is discussed. These regulators mostly use pole placement, sometimes with full pole cancellation. The other versions are based on characteristic polynomial design, which is a feedback regulator design method. Another class of the regulators are based on the Youla-parameterization, which is sometimes called Q-parameterization. Then it is shortly summarized how a Youla regulator is computed. Finally it is systematically proved that the best usable method is the Youla-parameterization based regulator design introduced by the authors.
\end{abstract}

Keywords: Regulator, Design, Performance, Parameterization

\section{Introduction}

The LTI state-space equations of a system generally applied in systems and control theory

$$
\begin{gathered}
\frac{\mathrm{d} \boldsymbol{x}(t)}{\mathrm{d} t}=\boldsymbol{A} \boldsymbol{x}(t)+\boldsymbol{b} u(t) \\
y(t)=\boldsymbol{c}^{\mathrm{T}} \boldsymbol{x}(t)+d_{\mathrm{c}} u(t)
\end{gathered}
$$

Here $u$ and $y$ are the input and output signals of the process, respectively, and $\boldsymbol{x}$ is the state vector. The parameter matrices of the system are $\boldsymbol{A}, \boldsymbol{b}, \boldsymbol{c}^{\mathrm{T}}, d$. Since this paper mainly treats $S I S O$ systems, in $n$-order case, matrix $\boldsymbol{A}$ means a $(n \times n)$ square matrix, which is the so-called state matrix, $\boldsymbol{b}$ is a column vector of $(n \times 1)$ size, $\boldsymbol{c}^{\mathrm{T}}$ is a row vector of $(1 \times n)$ size, and $d_{\mathrm{c}}$ is scalar [1].

The classical model of the dynamic LTI processes, the transfer function $P(s)$ is defined by the ratio of the LAPLACE transforms of the output and input signals, which can be easily derived from the state equation (1)

$$
P(s)=\frac{Y(s)}{U(s)}=\boldsymbol{c}^{\mathrm{T}}(s \boldsymbol{I}-\boldsymbol{A})^{-1} \boldsymbol{b}+d_{\mathrm{c}}=\frac{\mathcal{B}(s)}{\mathcal{A}(s)}
$$

where

$$
\begin{aligned}
& \mathcal{A}(s)=\operatorname{det}(s \boldsymbol{I}-\boldsymbol{A})=s^{n}+a_{1} s^{n-1}+\ldots+a_{n} \\
& \mathcal{B}(s)=b_{0} s^{m}+b_{1} s^{m-1}+\ldots+b_{m}
\end{aligned}
$$

The roots of the equation $\mathcal{A}(s)=0$ are called poles; the roots of $\mathcal{B}(s)=0$ are called zeros. A continuous-time (CT) linear process is stable, if all roots of the polynomial $\mathcal{A}(s)$ are located on the left-hand side of the complex plane. Concerning the order of the polynomials $\mathcal{A}(s)$ and $\mathcal{B}(s)$ it should be noted that the number of the state variables is $n$, $m$ is the order of the polynomial $\mathcal{B}(s)$, and the relation $m \leq n$ exists. The difference between the order of the numerator and denominator $p_{\mathrm{T}}=n-m$ is called pole access. If $p_{\mathrm{T}}>0$ then $P(s)$ is strictly proper, if $p_{\mathrm{T}}=0$ then the transfer function is proper. In the practice arbitrary relation $0 \leq p_{\mathrm{T}} \leq n$ might occur. 


\section{Basic Regulator Design Methods}

\subsection{Control Loops with State Feedback}

It was shown formerly how processes are represented in state-space. In many cases this kind of description is available only and the transfer function of the controlled system is unavailable. This partly explains why control design methodology directly based on state-space description has been evolved. Let us consider the state-space representation of an LTI process to be controlled such as

$$
\begin{aligned}
\frac{\mathrm{d} \boldsymbol{x}}{\mathrm{d} t} & =\dot{\boldsymbol{x}}=\boldsymbol{A} \boldsymbol{x}+\boldsymbol{b} u \\
y & =\boldsymbol{c}^{\mathrm{T}} \boldsymbol{x}
\end{aligned}
$$

which corresponds to (1) for the case of $d_{\mathrm{c}}=0$. This does not violate the generality, because it is very rare for the model to contain a proportional channel directly affecting the output. The block scheme of (4) and the classical statefeedback is shown in Figure 1, where the thick lines present vector variables and $r$ denotes the reference signal.

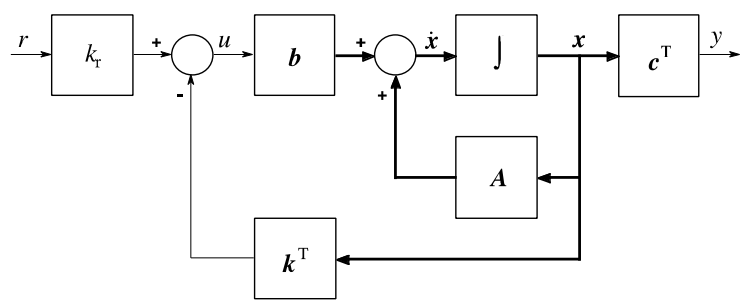

Figure 1. Linear regulator with state feedback.

In the closed-loop the state vector is fed back with the linear proportional vector $\boldsymbol{k}^{\mathrm{T}}$ according to the expression below

$$
u=k_{\mathrm{r}} r-\boldsymbol{k}^{\mathrm{T}} \boldsymbol{x}
$$

Based on Figure 1 the state equation of the complete closed system can be easily written as

$$
\begin{aligned}
\frac{\mathrm{d} \boldsymbol{x}}{\mathrm{d} t} & =\left(\boldsymbol{A}-\boldsymbol{b} \boldsymbol{k}^{\mathrm{T}}\right) \boldsymbol{x}+k_{\mathrm{r}} \boldsymbol{b} r \\
y & =\boldsymbol{c}^{\mathrm{T}} \boldsymbol{x}
\end{aligned}
$$

i.e., with the state feedback the dynamics represented by the original system matrix $\boldsymbol{A}$ is modified by the dyadic product $\boldsymbol{b} \boldsymbol{k}^{\mathrm{T}}$ to $\left(\boldsymbol{A}-\boldsymbol{b} \boldsymbol{k}^{\mathrm{T}}\right)$

The transfer function of the closed-loop control is

$$
\begin{aligned}
T_{\mathrm{ry}}(s) & =\frac{Y(s)}{R(s)}=\boldsymbol{c}^{\mathrm{T}}\left(s \boldsymbol{I}-\boldsymbol{A}+\boldsymbol{b} \boldsymbol{k}^{\mathrm{T}}\right)^{-1} \boldsymbol{b} \boldsymbol{k}_{\mathrm{r}}= \\
& =\frac{\boldsymbol{c}^{\mathrm{T}}(s \boldsymbol{I}-\boldsymbol{A})^{-1} \boldsymbol{b} \boldsymbol{k}_{\mathrm{r}}}{1+\boldsymbol{k}^{\mathrm{T}}(s \boldsymbol{I}-\boldsymbol{A})^{-1} \boldsymbol{b}}=\frac{k_{\mathrm{r}}}{1+\boldsymbol{k}^{\mathrm{T}}(s \boldsymbol{I}-\boldsymbol{A})^{-1} \boldsymbol{b}} P(s)=
\end{aligned}
$$

$$
=\frac{k_{\mathrm{r}} \mathcal{B}(s)}{\mathcal{A}(s)+\boldsymbol{k}^{\mathrm{T}} \boldsymbol{\Psi}(s) \boldsymbol{b}}
$$

which derives from the comparison of equations valid for the LAPLACE transforms, $U(s)=k_{\mathrm{r}} R(s)-\boldsymbol{k}^{\mathrm{T}} \boldsymbol{X}(s)$ (see (6)) and $Y(s)=c^{\mathrm{T}} \boldsymbol{X}(s)$ (see (4)) using the matrix inversion lemma. Note that the state feedback leaves the zeros of the process untouched and only the poles of the closed-loop system can be designed by $\boldsymbol{k}^{\mathrm{T}}$.

The so-called calibration factor $k_{\mathrm{r}}$ is introduced in order to make the gain of $T_{\text {ry }}$ equal to unity $\left(T_{\text {ry }}(0)=1\right)$. The open loop is obviously not of type one, so it cannot provide zero error and unity static transfer gain. It can be ensured only if the condition

$$
k_{\mathrm{r}}=\frac{-1}{\boldsymbol{c}^{\mathrm{T}}\left(\boldsymbol{A}-\boldsymbol{b} \boldsymbol{k}^{\mathrm{T}}\right)^{-1} \boldsymbol{b}}=\frac{\boldsymbol{k}^{\mathrm{T}} \boldsymbol{A}^{-1} \boldsymbol{b}-1}{\boldsymbol{c}^{\mathrm{T}} \boldsymbol{A}^{-1} \boldsymbol{b}}
$$

is fulfilled. The above special control loop is called state feedback [2, 3].

Pole placement by state feedback

The most natural design method of state feedback is the so-called pole placement. In this case the feedback vector $\boldsymbol{k}^{\mathrm{T}}$ needs to be chosen to make the characteristic equation of the closed-loop equal to the prescribed, so-called design polynomial $\mathcal{R}(s)$, i.e.,

$$
\begin{aligned}
\mathcal{R}(s) & =s^{n}+r_{1} s^{n-1}+\ldots+r_{n-1} s+r_{n}=\operatorname{det}\left(s \boldsymbol{I}-\boldsymbol{A}+\boldsymbol{b} \boldsymbol{k}^{\mathrm{T}}\right)= \\
& =\mathcal{A}(s)+\boldsymbol{k}^{\mathrm{T}} \boldsymbol{\Psi}(s) \boldsymbol{b}
\end{aligned}
$$

The solution always exists if the process is controllable. (It is reasonable if the order of $\mathrm{R}$ is equal to that of $\mathrm{A}$.) In the exceptional case when the transfer function of the controlled system is known, the canonical state equations can be directly written. Based on the controllable canonical form the system matrices are

$$
\begin{aligned}
& A_{\mathrm{c}}=\left[\begin{array}{ccccc}
-a_{1} & -a_{2} & \stackrel{-}{*} & -a_{n-1} & -a_{n} \\
1 & 0 & \rightleftharpoons & 0 & 0 \\
0 & 1 & & 0 & 0 \\
\vdots & \vdots & \ddots & \vdots & \vdots \\
0 & 0 & 0 & 1 & 0
\end{array}\right]
\end{aligned}
$$

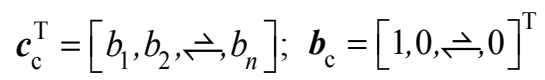

Considering the special forms of $\boldsymbol{A}_{\mathrm{c}}$ and $\boldsymbol{b}_{\mathrm{c}}$, it can be seen that the design equation (2.1.6) results in

$$
\boldsymbol{k}^{\mathrm{T}}=\boldsymbol{k}_{\mathrm{c}}^{\mathrm{T}}=\left[r_{1}-a_{1}, r_{2}-a_{2}, \boldsymbol{\nu}, r_{n}-a_{n}\right]
$$


ensuring the characteristic equation $(\mathcal{R}(s)=0)$, i.e., the prescribed poles. The choice of the calibration factor can be determined by simple calculation

$$
k_{\mathrm{r}}=\frac{a_{n}+\left(r_{n}-a_{n}\right)}{b_{n}}=\frac{r_{n}}{b_{n}}
$$

Based on equations (7), (8) and (9) it can be seen that in the case of state feedback pole placement the closed-loop transfer function results in

$$
T_{\mathrm{ry}}(s)=\frac{k_{\mathrm{r}} \mathcal{B}(s)}{\mathcal{R}(s)}
$$

The most common case of state feedback is when not the transfer function but the state-space form of the control system is given. It has to be observed that all controllable systems can be described in a controllable canonical form by using the transformation matrix $\boldsymbol{T}_{\mathrm{c}}=\boldsymbol{M}_{\mathrm{c}}^{\mathrm{c}}\left(\boldsymbol{M}_{\mathrm{c}}\right)^{-1}$. This linear transformation also refers to the feedback vector

$$
\begin{aligned}
& \boldsymbol{k}^{\mathrm{T}}=\boldsymbol{k}_{\mathrm{c}}^{\mathrm{T}} \boldsymbol{T}_{\mathrm{c}}=\boldsymbol{k}_{\mathrm{c}}^{\mathrm{T}} \boldsymbol{M}_{\mathrm{c}}^{\mathrm{c}} \boldsymbol{M}_{\mathrm{c}}^{-1} \\
& \boldsymbol{k}^{\mathrm{T}}=\boldsymbol{b}_{\mathrm{c}}^{\mathrm{T}} \boldsymbol{M}_{\mathrm{c}}^{-1} \mathcal{R}(\boldsymbol{A})=[0,0, \ldots, 1] \boldsymbol{M}_{\mathrm{c}}^{-1} \mathcal{R}(\boldsymbol{A})
\end{aligned}
$$

The design relating to the controllable canonical form (10), together with the linear transformation relationship corresponding to the first row of the non-controllable form (14), is known as the BASS-GURA algorithm [3]. The algorithm in the second row of (14) is called ACKERMANN method [9] after its elaborator.

In the Bass-Gura algorithm, the inverse of the controllability matrix $\boldsymbol{M}_{\mathrm{c}}$ needs to be determined by the general system matrices $\boldsymbol{A}$ and $\boldsymbol{b}$ on the one hand and the controllability matrix $\boldsymbol{M}_{\mathrm{c}}^{\mathrm{c}}$ of the controllable canonical form, on the other. Since this latter term depends only on the coefficients $a_{i}$ in the denominator of the process transfer function, the denominator needs to be calculated: $\mathcal{A}(s)=\operatorname{det}(s \boldsymbol{I}-\boldsymbol{A})$. Since $[0,0, \underset{\rightleftharpoons}{\rightleftharpoons} 1] \boldsymbol{M}_{\mathrm{c}}^{-1}$ is the last row of the inverse of the controllability matrix, and $\mathcal{R}(\boldsymbol{A})$ also need to be calculated; the Ackermann method does not need the calculation of $\mathcal{A}(s)$.

It is worth mentioning that the state feedback formally corresponds to a conventional $P D$ control and therefore overactuating peaks are expected at the input of the process because the pole placement tries to make the process faster. In practice, however, the actuator usually limits the amplitude of the peaks, which needs to be taken into account during the design of the poles of the characteristic polynomial $\mathcal{R}(s)$.

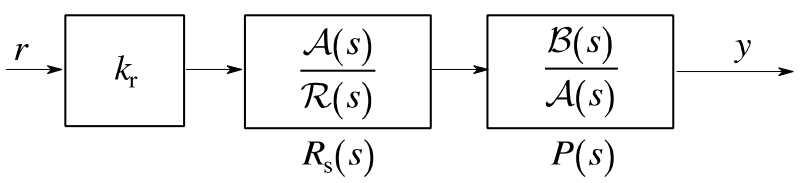

(a)

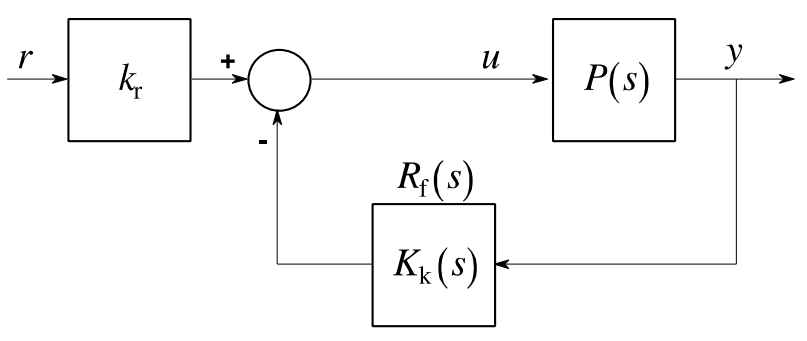

(b)

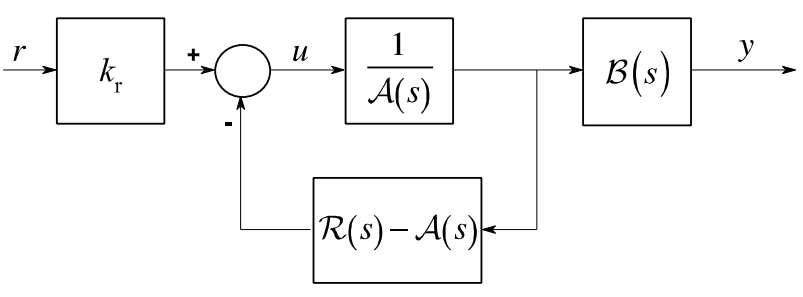

(c)

Figure 2. Equivalent schemes to the state feedback design using transfer functions and polynomials.

It can be clearly seen that state feedback formally corresponds to a serial compensation $R_{\mathrm{s}}=k_{\mathrm{r}} \mathcal{A}(s) / \mathcal{R}(s)$ (Figure 2a). The real operation and effect of the state feedback can be easily understood by the equivalent block schemes using the transfer functions shown in Figure 2. The "regulator" $R_{\mathrm{f}}(s)$ of the closed-loop is in the feedback line (Figure 2b). The transfer function of the closed-loop is

$$
\begin{aligned}
T_{\mathrm{ry}}(s) & =\frac{k_{\mathrm{r}} \mathcal{B}(s)}{\mathcal{R}(s)}=\frac{k_{\mathrm{r}} \mathcal{B}(s)}{\mathcal{A}(s)+\mathcal{B}(s)}=\frac{k_{\mathrm{r}} P(s)}{1+K_{\mathrm{k}}(s) P(s)}= \\
& =\frac{k_{\mathrm{r}} \mathcal{A}(s)}{\mathcal{R}(s)} \frac{\mathcal{B}(s)}{\mathcal{A}(s)}=k_{\mathrm{r}} R_{\mathrm{s}}(s) P(s)
\end{aligned}
$$

where

$$
R_{\mathrm{f}}=K_{\mathrm{k}}(s)=\frac{\mathcal{K}(s)}{\mathcal{B}(s)}=\frac{\mathcal{R}(s)-\mathcal{A}(s)}{\mathcal{B}(s)}=\frac{\boldsymbol{k}^{\mathrm{T}}(s \boldsymbol{I}-\boldsymbol{A})^{-1} \boldsymbol{b}}{\boldsymbol{c}^{\mathrm{T}}(s \boldsymbol{I}-\boldsymbol{A})^{-1} \boldsymbol{b}}
$$

and the calibration factor is

$$
k_{\mathrm{r}}=\frac{\boldsymbol{k}^{\mathrm{T}} \boldsymbol{A}^{-1} \boldsymbol{b}-1}{\boldsymbol{c}^{\mathrm{T}} \boldsymbol{A}^{-1} \boldsymbol{b}}=\frac{1+K_{\mathrm{k}}(0) P(0)}{P(0)}
$$

Given the block schemes of Figure 2 it can be stated that the state feedback also stabilizes the unstable terms, since due to the effect of the polynomial $\mathcal{K}(s)=\mathcal{R}(s)-\mathcal{A}(s)$ there 
is a pole placement for any process, so with the stable $\mathcal{R}(s)$ the stabilization is fulfilled. The feedback polynomial $\mathcal{K}(s)$ formally corresponds to $\boldsymbol{k}^{\mathrm{T}}$. The fact that the numerator $\mathcal{B}(s)$ of the process is present in the denominator of $K_{\mathrm{k}}(s)$ needs special consideration. The regulator can be applied only for minimum phase (inverse stable) processes, where the roots of $\mathcal{B}(s)$ are stable. As a consequence of this special character of the state feedback, however, here $\mathcal{B}(s)$ is not substituted by its model $\hat{\mathcal{B}}(s)$, but the method itself realizes the exact $1 / \mathcal{B}(s)$.

\subsection{Pole Placement with Pole Cancellation}

Consider the closed control system shown in Figure 3, where the regulator $C=\mathcal{A} / \mathcal{X}$ is used to place the poles of the closed control system according to the characteristic equation $\mathcal{R}=0$, ( $\mathcal{R}$ is the design polynomial) by the cancellation of the process poles. To do this, $\mathcal{X}$ needs to be expressed by the equation $\mathcal{R}=\mathcal{X}+\mathcal{B}$. The complementary sensitivity function of the closed-loop is:

$$
T=\frac{\frac{\mathcal{A}}{\mathcal{X}} \frac{\mathcal{B}}{\mathcal{A}}}{1+\frac{\mathcal{A}}{\mathcal{X}} \frac{\mathcal{B}}{\mathcal{A}}}=\frac{\mathcal{A B}}{\mathcal{A} \mathcal{X}+\mathcal{A B}}=\frac{\mathcal{B}}{\mathcal{X}+\mathcal{B}}=\frac{\mathcal{B}}{\mathcal{R}}
$$

The regulator is

$$
C=\frac{\mathcal{A}}{\mathcal{X}}=\frac{\mathcal{A}}{\mathcal{R}-\mathcal{B}}=\frac{\frac{\mathcal{B}}{\mathcal{R}}}{1-\frac{\mathcal{B}}{\mathcal{R}}} \frac{\mathcal{A}}{\mathcal{B}}=\frac{R_{\mathrm{r}}}{1-R_{\mathrm{r}}} P^{-1}
$$

and actually corresponds to an ideal Youla regulator with reference model $R_{\mathrm{r}}=R_{\mathrm{n}}=\mathcal{B} / \mathcal{R}$. This regulator places the poles in $\mathcal{R}$ and leaves the zeros in $\mathcal{B}$ untouched, if they are inverse stable.

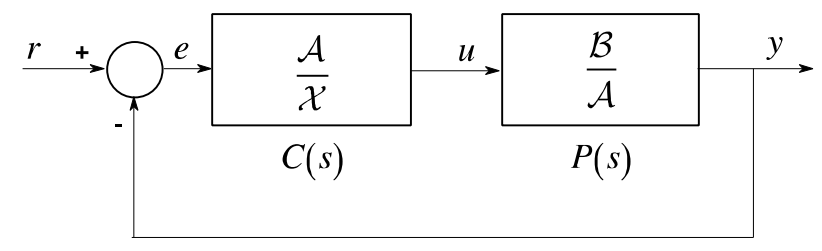

Figure 3. Pole canceling regulator.

\subsection{Pole Placement with Feedback Regulator}

An other solution when the regulator is put in the feedback is shown in Figure 4.

Now the task is again to place the poles of the closed system according to the characteristic equation $\mathcal{R}=0(\mathcal{R}$ is the design polynomial). To do this, $\mathcal{K}$ needs to be determined from the equation $\mathcal{R}=\mathcal{K}+\mathcal{A}$. The complementary sensitivity function of the closed system is

$$
T=\frac{\frac{\mathcal{B}}{\mathcal{A}}}{1+\frac{\mathcal{K}}{\mathcal{B}} \frac{\mathcal{B}}{\mathcal{A}}}=\frac{\mathcal{B}}{\mathcal{A}+\mathcal{K}}=\frac{\mathcal{B}}{\mathcal{R}}
$$

and thus this regulator places the poles in $\mathcal{R}$ and leaves the zeros in $\mathcal{B}$ untouched, if they are inverse stable.

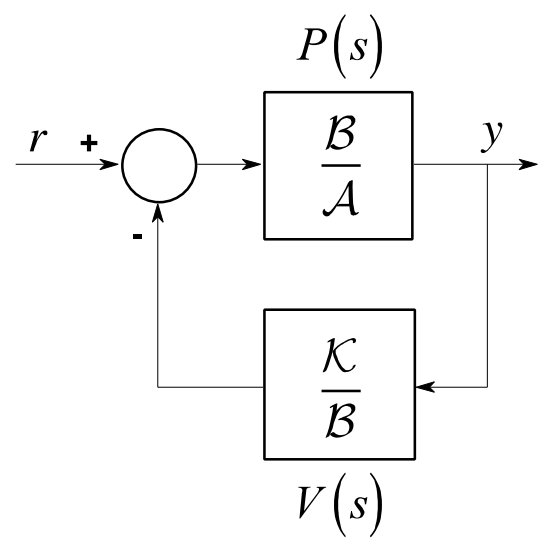

Figure 4. Regulator in the feedback.

The characteristic equation of the closed system has the form $\mathcal{R}=0$ and it doesnot depend on the unstable property of the process.

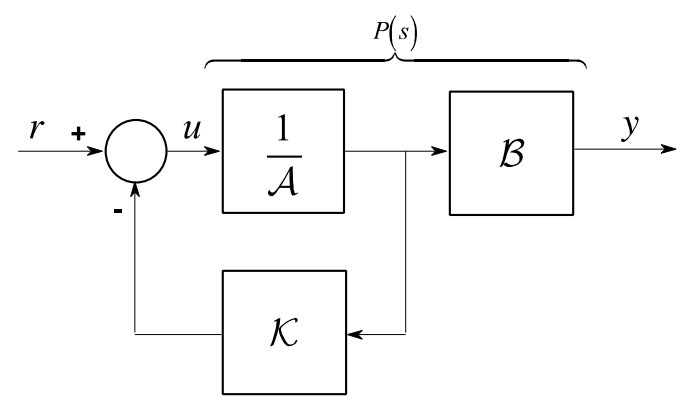

Figure 5. The regulator feeds back the internal signal of the process.

The block diagram in Figure 5. can be redrawn as Figure 2c. (The state feedback methods are discussed in detail in Section 2.1, and the same control principle is represented in Figure $2 \mathrm{c}$ among the schemes showing the equivalent transfer function representations for state feedback.)

\subsection{Pole Placement with Characteristic Polynomial Design}

The characteristic polynomial $\mathcal{R}$ of the closed-loop control can be directly designed by algebraic methods. In Figure 6 the regulator $C=\mathcal{Y} / \mathcal{X}$ is the quotient of two polynomials. Under certain conditions, the $D E \mathcal{A X}+\mathcal{B Y}=\mathcal{R}$ can be solved for $\mathcal{X}$ and $\mathcal{Y}$. Thus from the characteristic equation $\mathcal{R}=0$ the regulator can be directly determined. 


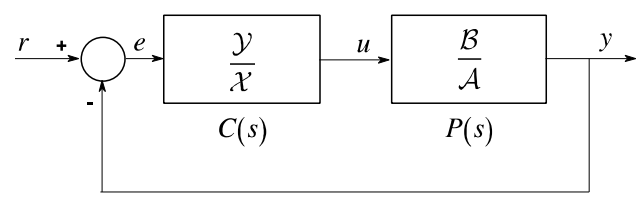

Figure 6. Direct control design on the basis of the characteristic polynomial.

The complementary sensitivity function of the closed system is

$$
T=\frac{\frac{\mathcal{Y}}{\mathcal{X}} \frac{\mathcal{B}}{\mathcal{A}}}{1+\frac{\mathcal{Y}}{\mathcal{X}} \frac{\mathcal{B}}{\mathcal{A}}}=\frac{\mathcal{B Y}}{\mathcal{A} \mathcal{X}+\mathcal{B Y}}=\frac{\mathcal{B Y}}{\mathcal{R}}
$$

and thus this regulator also places the poles in $\mathcal{R}$ and leaves the zeros in $\mathcal{B}$ untouched, but in the nominator $\mathcal{Y}$ appears, which depends on the desired properties and also on $D E$.

Thus the characteristic equation of the closed system has the form $\mathcal{R}=0$ and it does not depend on the unstable character of the process.

\subsection{Regulators Based on Youla Parameterization}

The Youla parameter, as a matter of fact, is a stable (by definition), regular transfer function

$$
Q(s)=\frac{C(s)}{1+C(s) P(s)} \text { or shortly } Q=\frac{C}{1+C P}
$$

where $C(s)$ is a stabilizing regulator, and $P(s)$ is the transfer function of the stable process [9].

It follows from the definition of the Youla parameter that the structure of the realizable and stabilizing regulator in the Youla-parameterized control loop is fixed:

$$
C(s)=\frac{Q(s)}{1-Q(s) P(s)} \text { or shortly } C=\frac{Q}{1-Q P}
$$

The sensitivity and complementary sensitivity functions linear in $Q$ of the closed control systems [10] were defined by (25). It is interesting to observe that the $Y P$ regulator [11] of (23) can be realized by a simple control loop with positive feedback as shown in Figure 7.

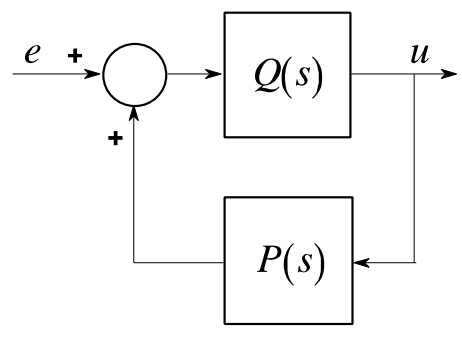

Figure 7. Realization of YP regulator.

A Youla-parameterized $(Y P)$ (sometimes called $Q$ parameterized) closed-loop is shown in Figure 8.

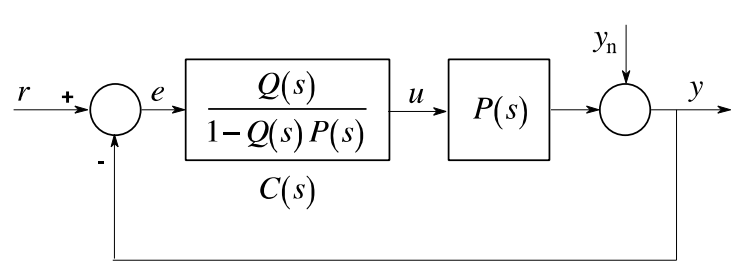

Figure 8. Youla-parametrized closed-loop.

The All-Realizable-Stabilizing (ARS) regulator has the form of (23).

The closed-loop transfer function or Complementary Sensitivity Function (CFS)

$$
T=\frac{C P}{1+C P}=Q P
$$

which is linear in the Youla parameter $Q$. It is well known that the $Y P$ regulator corresponds to the classical $I M C$ (Internal Model Control) structure.

The relationships between the most important signals of the closed system can be obtained with simple calculations

$$
\begin{aligned}
& u=Q r-Q y_{\mathrm{n}} \\
& e=(1-Q P) r-(1-Q P) y_{\mathrm{n}}=S r-S y_{\mathrm{n}} \\
& y=Q P r+(1-Q P) y_{\mathrm{n}}=T r+S y_{\mathrm{n}}
\end{aligned}
$$

The effect of $r$ and $y_{\mathrm{n}}$ on $u$ and $e$ is completely symmetrical (not considering the sign). Thus the input of the process depends only on the external signals and $Q(s)$.

From the equation (24) it can be seen that the Youla parameterization has the transfer function $Q P r$ concerning the reference signal tracking. If the $K B$ parameterization [4] is introduced on the figure of Figure 8, then the Youla parameterization can be simply extended for TDOF control systems. To do this, let us simply apply a parameter $Q_{\mathrm{r}}$ for the design of the tracking properties, and connect it in serial to the $K B$-parameterized loop, so the block diagram of Figure 9 is obtained.

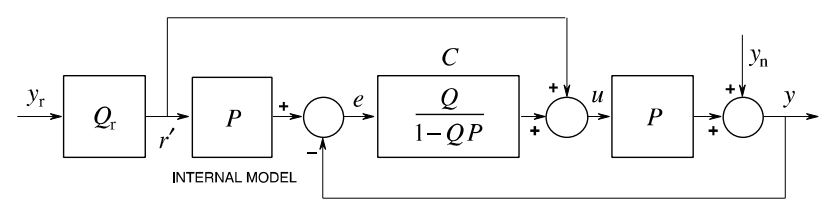

Figure 9. Two-degree-of-freedom version of the YP control loop.

The overall transfer characteristics for this system are

$$
\begin{aligned}
& u=Q_{\mathrm{r}} y_{\mathrm{r}}-Q y_{\mathrm{n}} \\
& e=\left(1-Q_{\mathrm{r}} P\right) y_{\mathrm{r}}-(1-Q P) y_{\mathrm{n}}=\left(1-T_{\mathrm{r}}\right) y_{\mathrm{r}}-S y_{\mathrm{n}} \\
& y=Q_{\mathrm{r}} P y_{\mathrm{r}}+(1-Q P) y_{\mathrm{n}}=T_{\mathrm{r}} y_{\mathrm{r}}+(1-T) y_{\mathrm{n}}=T_{\mathrm{r}} y_{\mathrm{r}}+S y_{\mathrm{n}}
\end{aligned}
$$

where the tracking properties can be designed by choosing $Q_{\mathrm{r}}$ in $T_{\mathrm{r}}=Q_{\mathrm{r}} P$, and the noise rejection properties by 
choosing $Q$ in $T=Q P$. These two properties can be handled separately. The reference signal of the whole system is denoted by $y_{\mathrm{r}}$. The conditions for $Q_{\mathrm{r}}$ are the same as for $Q$. The meaning of $T_{\mathrm{r}}$ is analogous to the meaning of the complementary sensitivity function $T$ of the one-degree-offreedom control loop for tracking $[5,6]$.

\section{Comparision of the Previously Discussed Design Methods}

\section{Control loops with state feedback}

The most important advantage of the state feedback regulator, is that the calculation of the feedback vector is very simple. The most important disadvantage is that the internal state variables, necessary for the feed-back are usually not available in the practical tasks. This is why the observer topology is generally necessary to this method. Unfortunately this topology is not so simply to compute. Another important disadvantage is that this regulator assigns the pole of the closed-loop system, unfortunately it leaves the numerator of the process untouched in $T$. It is important to know that from the methods discussed in this paper this is the only method which is applicable for unstable processes.

Pole placement with pole cancellation

The most important advantage of this method that it is very simple to calculate the regulator. The disadvantage is that this regulator assigns the pole of the closed-loop system, unfortunately it also leaves the numerator of the process untouched in $T$.

\section{Pole placement with feedback regulator}

This method practically can be evaluated on the similar way as the previous method. Unfortunately the most important disadvantage is that in a practical task it is very rare that the regulator is in the feedback line.

Pole placement with characteristic polynomial design

This method is a little bit more complex than the pole cancellation method, because the calculation of the regulator needs the solution of a $D E$. The disadvantage is that this regulator assigns the pole of the closed-loop system, unfortunately it also leaves the numerator of the process untouched in $T$ and puts another polynomial in the numerator of $T$. This polynomial comes from the solution of the $D E$, so it is not easy to design.

\section{Regulators based on Youla parameterization}

This method is the simplest, because it needs only basic polynomial operations to calculate the regulator. A further advantage is that the result of the design is the best reachable $T$ even for invariant process zeros, too.

Except the state feedback regulator the other methods are applicable only for stable processes.

\section{Computation of the Optimal Youla Regulator}

Let us assume the transfer function of the process in the following factorized form

$$
P(s)=P_{+}(s) \bar{P}(s)_{-}=P_{+}(s) P_{-}(s) e^{-s T_{\mathrm{d}}}
$$

or shortly

$$
P=P_{+} \bar{P}_{-}=P_{+} P_{-} e^{-s T_{\mathrm{d}}}
$$

where $P_{+}$is stable, and its inverse is also stable (Inverse Stable: IS) and realizable (ISR). The inverse of $\bar{P}_{-}$is unstable (Inverse Unstable: IU) and not realizable (Non Realizable: NR), i.e., (IUNR). $P_{-}$is inverse unstable (IU).

Here, in general, the inverse of the dead-time part $e^{-s T_{\mathrm{d}}}$ is not realizable, because it would be an ideal predictor.

In polynomial form a delay free process is given by

$$
P(s)=\frac{\mathcal{B}(s)}{\mathcal{A}(s)}=\frac{\mathcal{B}_{+}(s) \mathcal{B}_{-}(s)}{\mathcal{A}(s)}
$$

where $\mathcal{B}_{+}(s)$ and $\mathcal{B}_{-}(s)$ contain the inverse stable and inverse unstable zeros, respectively.

If the reference model, formulating our design goal is

$$
R_{\mathrm{n}}(s)=\frac{\mathcal{B}_{\mathrm{n}}(s)}{\mathcal{A}_{\mathrm{n}}(s)}
$$

then the optimal Youla parameter is

$$
Q(s)=R_{\mathrm{n}}(s) \mathcal{B}_{+}^{-1}(s)
$$

Using this parameterization the optimal Youla regulator can be calculated as $[7,8]$

$$
\begin{aligned}
C(s) & =\frac{Q(s)}{1-Q(s) P(s)}=\frac{R_{\mathrm{n}}(s) \mathcal{B}_{+}^{-1}(s)}{1-R_{\mathrm{n}}(s) \mathcal{B}_{+}^{-1}(s) \mathcal{B}_{+}(s) \mathcal{B}_{-}(s)}= \\
& =\frac{\mathcal{B}_{\mathrm{n}}(s) \mathcal{A}(s)}{\mathcal{B}_{+}(s)\left[\mathcal{A}_{\mathrm{n}}(s) \mathcal{A}(s)-\mathcal{B}_{\mathrm{n}}(s) \mathcal{B}_{-}(s)\right]}
\end{aligned}
$$

The transfer function of the closed-loop system is

$$
T(s)=R_{\mathrm{n}}(s) \mathcal{B}_{-}(s)=\frac{\mathcal{B}_{\mathrm{n}}(s)}{\mathcal{A}_{\mathrm{n}}(s)} \mathcal{B}_{-}(s)
$$

which is the best reachable result for the case of inverse unstable zeros. This result explains the name: "uncancellable" for the inverse unstable factors of the numerator of the process.

For the two-degree-of-freedom version of the Youla regulator (see Figure 9) an additional reference model

$$
R_{\mathrm{r}}(s)=\frac{\mathcal{B}_{\mathrm{r}}(s)}{\mathcal{A}_{\mathrm{r}}(s)}
$$


must also be calculated.

It can be well seen in this section that the computation of the Youla regulator requires only very simple polynomial operations (additions and multiplications).

Example 4.1. Let the CT process be given by a nonminimum phase transfer function

$$
P(s)=\frac{\left(1+s \tau_{1}\right)\left(1-s \tau_{2}\right)}{\left(1+s T_{1}\right)\left(1+s T_{2}\right)\left(1+s T_{3}\right)}
$$

where $T_{1}=10 \mathrm{sec} ; T_{2}=5 \mathrm{sec} ; T_{3}=2 \mathrm{sec} ; \tau_{1}=6 \mathrm{sec}$ and $\tau_{2}=4 \mathrm{sec}$, where $\mathcal{B}_{+}=\left(1+s \tau_{1}\right)$ and $\mathcal{B}_{-}=\left(1-s \tau_{2}\right)$.

The selected reference model is

$$
R_{\mathrm{n}}(s)=\frac{\mathcal{B}_{\mathrm{n}}(s)}{\mathcal{A}_{\mathrm{n}}(s)}=\frac{1+s \tau_{\mathrm{n} 1}}{1+s T_{\mathrm{n} 1}}=\frac{1}{1+s T_{\mathrm{n} 1}}
$$

where $T_{\mathrm{n} 1}=5 \mathrm{sec} ; \tau_{\mathrm{n} 1}=0$.

The optimal Youla regulator can be calculated as

$$
\begin{aligned}
& C(s)=\frac{\mathcal{B}_{\mathrm{n}}(s) \mathcal{A}(s)}{\mathcal{B}_{+}(s)\left[\mathcal{A}_{\mathrm{n}}(s) \mathcal{A}(s)-\mathcal{B}_{\mathrm{n}}(s) \mathcal{B}_{-}(s)\right]}= \\
& =\frac{\left(1+s T_{1}\right)\left(1+s T_{2}\right)\left(1+s T_{3}\right)}{\left(1+s \tau_{1}\right)\left[\left(1+s T_{\mathrm{n} 1}\right)\left(1+s T_{1}\right)\left(1+s T_{2}\right)\left(1+s T_{3}\right)-\left(1-s \tau_{2}\right)\right]}
\end{aligned}
$$

Using the numerical values the regulator is

$$
C(s)=\frac{1+17 s+80 s^{2}+100 s^{3}}{s(1+6 s)\left(1+2.273 s+4.454 s^{2}\right)}
$$

The overall transfer function of the closed-loop system is

$$
T(s)=R_{\mathrm{n}}(s) \mathcal{B}_{-}(s)=\frac{1-s \tau_{2}}{1+s T_{\mathrm{n} 1}}=\frac{1-4 s}{1+5 s}
$$

Because usually the reference model has unity gain, i.e.

$$
\mathcal{B}_{\mathrm{n}}(0)=\mathcal{A}_{\mathrm{n}}(0)
$$

it follows, that $T(0)=1$ has also unity gain.

The usual normalization of the process polynomial means that $\mathcal{A}(0)=1$ and $\mathcal{B}_{-}(0)=1$ (while $\mathcal{B}_{+}(0) \neq 1$ ) it can be easily checked that the Youla regulator is always an integrating regulator for (40).

Example 4.2. Investigate now a discrete-time (DT) case, when the pulse transfer function of the process is a second order system

$$
P(z)=\frac{-0.32(z-1.25)}{(z-0.8)(z-0.6)}
$$

and the reference model is

$$
R_{\mathrm{n}}(z)=\frac{0.6}{z-0.4}
$$

The optimal Youla regulator can be computed now as

$$
C(z)=\frac{-0.624\left(1-2.917 z+2.083 z^{2}\right)}{1+0.027 z-1.248 z^{2}+0.693 z^{3}}
$$

\section{Conclusions}

It was shown that the Youla regulator design is a very simple procedure, which is applicable for all kind of (minimum or non minimum phase) CT and DT processes. The computation of the regulator is very simple, requires only polynomial operations.

For reasonable design goal this design results in an integrating regulator.

This regulator ensures the theoretical best reachable closed-loop property of the control system.

\section{References}

[1] Åström, K. J. and B. Wittenmark (1984). Computer Controlled Systems. Prentice-Hall, p. 430.

[2] Goodwin, G. C., Graebe S. F. and Salgado M. E. (2001). Control System Design. Prentice-Hall, p. 908.

[3] Horowitz, I. M. (1963). Synthesis of Feedback Systems, Academic Press, New York.

[4] Keviczky, L. (1995). Combined identification and control: another way. (Invited plenary paper.) 5th IFAC Symp. on Adaptive Control and Signal Processing, ACASP'95, 13-30, Budapest, Hungary.

[5] Keviczky, L. and Cs. Bányász (1999). Optimality of twodegree of freedom controllers in $\mathrm{H}_{2}$ - and $\mathrm{H}_{\infty}$-norm space, their robustness and minimal sensitivity. 14th IFAC World Congress, F, 331-336, Beijing, PRC.

[6] Keviczky, L. and Cs. Bányász (2015). Two-Degree-ofFreedom Control Systems (The Youla Parameterization Approach), Elsevier, Academic Press, p. 512.

[7] Keviczky, L., R. Bars, J. Hetthéssy and Cs. Bányász (2018). Control Engineering. Springer.

[8] Keviczky, L., R. Bars, J. Hetthéssy and Cs. Bányász (2018). Control Engineering: MATLAB Exercises, Springer.

[9] Maciejowski, J. M. (1989). Multivariable Feedback Design, Addison Wesley, p. 424.

[10] Youla, D. C., Bongiorno, J. J. and C. N. Lu (1974). Singleloop feedback stabilization of linear multivariable dynamical plants, Automatica, Vol. 10, 2, pp. 159-173.

[11] Youla, D. C. and J. J. Bongiorno, Jr. (1985). A feedback theory of two-degree-of-freedom optimal Wiener-Hopf design," IEEE Trans. Auto. Control, vol. AC-30, No. 7, pp. 652-665. 